\section{Neues raumtemperaturstabiles NovoSeven ${ }^{\circledR}$ : Mehr Therapiekom- fort für Hämophilie-Patienten}

NovoSeven ${ }^{\circledR}$, das einzige rekombinante aktivierte Faktor-VII-Produkt (rFVIIa) zur Behandlung von Blutungsepisoden bei Patienten mit Hemmkörper-Hämophilie, liegt ab Februar 2009 als raumtemperaturstabile Formulierung vor. Dies gab das Unternehmen Novo Nordisk Pharma GmbH bekannt. Das Präparat ist dadurch jederzeit verfügbar und ermöglicht eine sofortige Behandlung von Blutungen. Dies bedeutet einen erhöhten Therapiekomfort und damit eine deutliche Verbesserung der Lebensqualität für die Patienten. Die neue raumtemperaturstabile Formulierung von NovoSeven ${ }^{\circledR}$ bietet den Vorteil, dass sie jederzeit verfügbar ist. Dies gibt dem Patienten die Möglichkeit,
NovoSeven ${ }^{\circledR}$ im Fall einer Blutung jederzeit auch unterwegs - rasch und effektiv anwenden zu können. Die raumtemperaturstabile Formulierung ersetzt die bisherige Formulierung von NovoSeven ${ }^{\circledR}$. Das raumtemperaturstabile NovoSeven ${ }^{\circledR}$ ist bei Raumtemperatur (bis zu $25^{\circ} \mathrm{C}$ ) bis zu 2 Jahre stabil [1]. Das neue Präparat ist bioäquivalent und besitzt vergleichbare pharmakokinetische Eigenschaften wie die Vorgängerformulierung [2]. Da die Konzentration der rekonstituierten Lösung bei der raumtemperaturstabilen Formulierung im Vergleich zur Vorgänger-Formulierung erhöht ist, ist das Injektionsvolumen bei dem neuen Präparat um $40 \%$ reduziert. Ein weiterer Vorteil: Die Berechnung des Injektionsvolumens wird erleichtert, da das neue rFVIIa-Präparat nun in ganzzahligen Dosisstärken von 1, 2 und $5 \mathrm{mg}$ vorliegt. $\mathrm{Zu}$ dem raumtemperaturstabilen NovoSeven ${ }^{\circledR}$ Präparat gibt es zusätzlich eine tragbare, stabile
Reise-Box, in der alle Utensilien für die Behandlung einer einzelnen Blutung unterwegs zur Verfügung stehen. Die Raumtemperaturstabilität ermöglicht einen sofortigen Therapiebeginn, sodass Blutungen schnell gestoppt werden können [3].

\section{Referenzen}

1 NovoSeven ${ }^{\circledR}(1 \mathrm{mg}(50 \mathrm{kIE}) / 2 \mathrm{mg}(100 \mathrm{kIE}) / 5 \mathrm{mg}(250$ kIE)) Fachinformation, Stand: September 2008.

2 Bysted BV et al: Haemophilia 2007;13:527-532.

3 Lusher JM. Blood Coagul Fibrinolysis 2000;11(Suppl 1):45-49.

Weitere Informationen bei

Medizin \& Markt GmbH

Franziska Wagenschwanz

Schackstraße 1/IV, 80539 München

Tel.+49 89 383930-22, Fax +49 89339075

wagenschwanz@medizin-und-markt.de

\section{Erstmals interaktiver Workshop zur Hämophilie-Therapie: Frühe Behandlung entscheidend}

Eine frühe prophylaktische Therapie mit dem fehlenden Gerinnungsfaktor kann das Hemmkörperrisiko sowie die Anzahl der Blutungen bei Hämophilie-Patienten deutlich reduzieren. Dies zeigen Studiendaten [1, 2], die auf einem interaktiven Computer-gestützten Workshop von Wyeth zur Hämophilietherapie in Berlin vorgestellt wurden. Ein weiterer wichtiger Aspekt des Workshops war die Bedeutung der Publikation neuer Daten in der Hämophilie. Gemeinsamer Konsens von jungen und erfahrenen Hämophilie-Behandlern beim Workshop: Um langfristig die Hämophilietherapie weiter zu optimieren, sollten Pharmakovigilanz-Unter- suchungen und andere Erhebungen unterstützt werden. Für die rekombinanten Produkte ReFacto ${ }^{\circledR}$ (Moroctocog alfa) und BeneFIX $^{\circledR}$ (Nonacog alfa) von Wyeth gibt es bereits solche nicht-interventionellen Studien.

Der 2-tätige Workshop fand in diesem Jahr erstmals in dieser Form statt und wurde von der Ärztekammer Berlin mit 10 CME-Punkten zertifiziert. Aufgegriffen wurden verschiedene Aspekte der Hämophilietherapie sowie Herausforderungen des Praxisalltags in Vorträgen und interaktiven Falldiskussionen. In der 2-tägigen Veranstaltung trugen insgesamt 17 Referenten dazu bei, in Frontalvorträgen verschiedene Themenblöcke der Hämophilie zu beleuchten. Die anschließenden interaktiven Falldiskussionen mit TEDAbstimmung förderten die fachliche Diskussion und einen regen Austausch unter den
Experten. Der Workshop fand in diesem Jahr erstmals in dieser Computer-gestützten Form statt, soll aber auch interaktiv fortgeführt werden.

\section{Referenzen}

1 Gouw S, van der Bom J, van den Berg: Treatment-related risk factors of inhibitor development in previously untreated patients with hemophilia A: The CANAL cohort study. Blood 2007; 109:4648-4654.

2 Manco-Johnson $\mathrm{M}$ et al.: Prophylaxis versus episodic treatment to prevent joint disease in boys with severe hemophilia. N Engl J Med 2007;357:535-54.

Weitere Informationen bei

Wyeth Pharma GmbH

Pressestelle, Mirja Kaupmann

Wienburgstraße 207, 48159 Münster

Tel.+49 251 204-1226, Fax -2033

www.wyeth.de

\title{
Ticker+++ Ticker+++ Ticker+++ Ticker+++ Ticker+++ Ticker+++ Ticker+++
}

Leo Pharma GmbH. Tumorpatienten haben ein überproportional erhöhtes Risiko für venöse Thromboembolien (VTE). Fachgesellschaften empfehlen deshalb zur perioperativen Thromboseprophylaxe und zur medikamentösen Langzeittherapie nach einer VTE den Einsatz eines niedermolekularen Heparins. Mittel der Wahl bei diesen Risikopatienten ist Tinzaparin (innohep ${ }^{\circledR}$ ).

Wefra PR Gesellschaft für Public Relations mbH

Judith Schönberger

Tel. +49 69 695008-76

js@wefra.de
Mundipharma GmbH. Als First-line-Therapie von indolenten NHL und Mantelzell-Lymphomen war Bendamustin + Rituximab (BR) in einer Studie ebenso effektiv wie CHOP-R, bei besserer Verträglichkeit. Die Phase-II-Studie CLL2M untersuchte BR auch bei rezidivierter/refraktärer und unvorbehandelter CLL. Um den Stellenwert von BR als First-line-Therapie der CLL weiter zu definieren, wird es nun mit Fludarabin + Cyclophosphamid + Rituximab (FCR) verglichen (Studie CLL10).

POMME-med GmbH Dr.Petra Ortner ortner@pomme-med.de
Alexion Pharma Germany GmbH. Der rekombinante monoklonale Antikörper Soliris ${ }^{\circledR}$ (Eculizumab) greift kausal in den Pathomechanismus der paroxysmalen nächtlichen Hämoglobinurie $(\mathrm{PNH})$ ein. Die Erkrankung führt zu einem erhöhten Risiko für lebensbedrohliche thromboembolische Komplikationen. Mit der deutlichen Verbesserung der Beschwerden und der Reduktion von thromboembolischen Ereignissen hat Eculizumab den Verlauf der PNH maßgeblich verändert und neue Maßstäbe für die Behandlung gesetzt.

Alexion Pharma Germany GmbH

Melanie Hansen

hansenm@alxn.com 